\title{
Endoscopic vs open saphenous vein harvest for coronary artery bypass grafting: A leg-related morbidity and histological comparison
}

\author{
Radim Brata , Jaroslav Horacek ${ }^{b}$, Jiri Sieja ${ }^{\mathrm{a}}$
}

\begin{abstract}
Aims. The aim of this study was to compare coronary artery bypass grafting (CABG) using either endoscopic (EVH) or open harvest $(\mathrm{OVH})$. Leg-related morbidity and histological comparison of the veins were the outcome measures. Methods. One hundred consecutive patients scheduled for isolated CABG were randomly divided into two goups: an $\mathrm{EVH}$ and OVH group. Perioperative data were recorded. Patients were examined 7 days and 1 month postoperatively for leg-related morbidity. Samples for histological examination were taken from each harvested vein during the surgery. Results. Postoperative pain was statistically significantly lower in the EVH group 7 days postoperatively but the incidence of haematoma was non statistically higher in the EVH group while swelling was higher in the OVH group. Almost $40 \%$ of all histological samples were described as showing endothelial damage. There was significantly more endothelial damage in the $\mathrm{EVH}$ than the $\mathrm{OVH}$ group.

Conclusions. We confirmed the advantage of EVH in terms of leg-related morbidity as well as cosmetic effect. This method however, was associated with more acute endothelial damage of the graft. These results support concerns that endoscopic vein harvest may be connected with detrimental effects on vein endothelium which could promote a thrombogenic environment leading to a decrease in graft patency. This could be extremely important. The results suggest that further investigation of the long-term patency of vein grafts harvested endoscopically is required.
\end{abstract}

Key words: saphenous vein harvest, edoscopic harvest, histopathology, endothelial damage, minimally invasive, coronary artery bypass grafting

Received: October 27, 2011; Accepted with revision: January 12, 2012; Available online: January 31, 2012 http://dx.doi.org/10.5507/bp.2012.011

${ }^{a}$ Cardiosurgical Department, University Hospital Ostrava and Faculty of Medicine, University of Ostrava, Czech Republic bInstitute of Pathology, Faculty of Medicine, University of Ostrava

Corresponding author: Radim Brat, e-mail: radim.brat@fno.cz

\section{INTRODUCTION}

Coronary Artery Bypass Grafting (CABG) continues to be a widely used therapy for the treatment of ischemic heart disease and it remains the most common procedure in cardiothoracic surgery ${ }^{1}$. Despite advances in the use of arterial grafts, the long saphenous vein remains the most commonly used conduit for coronary artery bypass surgery ${ }^{2}$.

Traditional methods of vein harvest, in which a wound is opened along the length of the long saphenous vein, often contribute significantly to patient morbidity ${ }^{3,4}$. Minimally invasive techniques of vein harvest have been developed in order to reduce this significant morbidity. Studies have shown reduced rates of postoperative wound complications following minimally invasive approaches compared to the traditional saphenous vein harvest technique ${ }^{5,6}$. Despite these studies, however, there is a paucity of data on the mid- term and long-term results. Also the histological data published in the literature have given rise to a mixed opinion ${ }^{7,8}$.

The aim of this study was to examine local outcomes with endoscopic vein harvest $(\mathrm{EVH})$ and to provide more data on the histological comparison of the veins after endoscopic and open harvest.

\section{MATERIALS AND METHODS}

From October 2009 to August 2010, 100 consecutive patients scheduled for isolated CABG using the great saphenous vein graft and meeting the inclusion criteria were randomly divided into 2 groups. EVH was used in the $1^{\text {st }}$ group and $\mathrm{OVH}$ was used in the $2^{\text {nd }}$ group. The study was performed as a prospective randomized study. All patients included in this study were acceptable for both saphenous vein harvest methods - open as well as endoscopic. Informed consent was obtained from all patients and the study was approved by the ethics committee.

Ultrasound mapping of both great saphenous veins using the vascular $10 \mathrm{MHz}$ probe was done before the surgery. Only patients with a patent deep vein system, location of the great saphenous vein at least $4 \mathrm{~mm}$ under the skin surface, lumen of the vein from 2,0 to $5,0 \mathrm{~mm}$ and vein wall thickness less than $1,5 \mathrm{~mm}$ were included in the study.

Patients were randomized into two groups - open vein harvest group (OVH group) and endoscopic harvest group (EVH group). In the OVH group the graft was harvested by an advanced surgeon using standard open technique with ligating or clipping the side branches. In the EVH group the endoscopy was performed by a sur- 
geon with sufficient experience in this harvest method. A standardized miniinvasive technique was performed using the Virtuo-Saph (Terumo) endoscopic vessel harvesting system and carbon dioxide insufflation technique. Diathermy was employed to divide side branches in situ with titanium clips applied prior to grafting.

Important perioperative data were recorded. In the follow-up, patients were examined 7 days and 1 month after the surgery focusing on leg-related morbidity. Leg wound pain was recorded as patient subjective feeling and complaints of pain of any severity. One sample for histological examination was taken from each harvested vein during the surgery. The sample of at least $10 \mathrm{~mm}$ was taken from the distal part of the vein. The biopsy material was processed using the paraffin technique with basic staining and immunohistochemistry assay. Histological changes were recorded. Assessment of the changes was contributed to by immunohistochemistry assay using Factor VIII, CD34, HAS and Laminin antibodies and by Trichrome and VG chemical stains.

Comparison between groups was made with Student's t-test when appropriate. Values of $P<0.001$ were considered statistically significant.

\section{RESULTS}

The clinical characteristics of the patients are summarized in Table 1. There was no significant difference between groups in the clinical profile. The majority of the patients were male and there was a high proportion of diabetics in both groups. Perioperative data describing the vein harvest are shown in Table 2. There was no difference in the mean length of the graft harvested in either group. Also the difference in the harvest time between groups was not statistically significant. Statistically significant was the difference in number of tears and branches sutured using 7-0 Prolene (higher in the EVH group) and first of all in the overall length of skin incisions, which was of course much shorter in the EVH group. Data describing leg-related morbidity 7 days and 1 month postoperatively are shown in Table 3, 4. Data were analysed in all patients. We can see, that there is a statistically significant difference in the postoperative pain between the groups 7 days postoperatively. The pain was lower in the EVH group. On the other hand, EVH was connected with higher incidence of haematoma, but this difference did not reach a statistical significance and nor

Table 1. Clinical characteristics of the patients.

\begin{tabular}{lccc}
\hline & EVH group & OVH group & $P$-value \\
\hline No. of patients & 50 & 50 & NS \\
Male (\%) & $42(84 \%)$ & $45(90 \%)$ & NS \\
Female (\%) & $8(16 \%)$ & $5(10 \%)$ & NS \\
Age (years) & $65 \pm 8.94$ & $66.2 \pm 8.17$ & NS \\
LVEF $(\%)$ & $53.4 \pm 8.85$ & $52.4 \pm 9.09$ & NS \\
AP $(\mathrm{CCS})$ & $1.7 \pm 1.0$ & $2.3 \pm 1.02$ & NS \\
BMI $\left(\mathrm{kg} / \mathrm{m}^{2}\right)$ & $29.1 \pm 4.0$ & $28.9 \pm 4.08$ & NS \\
Diabetics $(\%)$ & $20(40 \%)$ & $22(44 \%)$ & NS \\
Smokers $(\%)$ & $18(36 \%)$ & $16(32 \%)$ & NS \\
Smokers and diabetics (\%) & $6(12 \%)$ & $5(10 \%)$ & NS \\
\hline
\end{tabular}

Where applicable, data are shown as the mean \pm the standard deviation.

LVEF...left ventricular ejection fraction

AP...angina

BMI... body mass index

NS... not significant $(P>0.05)$

Table 2. Perioperative data.

\begin{tabular}{lccc}
\hline & EVH group & OVH group & $P$-value \\
\hline No. of patients & 50 & 50 & NS \\
Length of the graft $(\mathrm{cm})$ & $36.9 \pm 8.35$ & $38.4 \pm 11.23$ & NS \\
Harvest time $(\mathrm{min})$ & $36.2 \pm 13.25$ & $37.7 \pm 20.46$ & NS \\
No. of conversions & 0 & NA & \\
No. of 7-0 sutures & $1.3 \pm 1.57$ & $0.4 \pm 0.94$ & $P<0.001$ \\
Overall length of skin incisions $(\mathrm{cm})$ & $7.6 \pm 3.1$ & $40.3 \pm 12.81$ & $P<0.001$ \\
\hline
\end{tabular}

Where applicable, data are shown as the mean \pm the standard deviation.

NS... not significant $(P>0.05)$ 
Table 3. Leg-related morbidity 7 days postoperatively.

\begin{tabular}{lccc}
\hline & EVH group & OVH group & $P$-value \\
\hline No. of patients & 50 & 50 & NS \\
Haematoma (\%) & $31(62 \%)$ & $22(44 \%)$ & NS \\
Leg wound dehiscence (\%) & 0 & 0 & NS \\
Swelling (\%) & $4(8 \%)$ & $13(26 \%)$ & NS \\
Leg wound infection (\%) & 0 & 0 & NS \\
Necrosis (\%) & 0 & $1(2 \%)$ & NS \\
Leg wound pain (\%) & $6(12 \%)$ & $22(44 \%)$ & $P<0.001$ \\
Dysaestesia (\%) & 0 & $5(10 \%)$ & NS \\
\hline
\end{tabular}

NS... not significant $(P>0.05)$

Table 4. Leg-related morbidity 1 month postoperatively.

\begin{tabular}{lccc}
\hline & EVH group & OVH group & $P$-value \\
\hline No. of patients & 50 & 50 & NS \\
Haematoma (\%) & $1(2 \%)$ & 0 & NS \\
Leg wound dehiscence (\%) & $1(2 \%)$ & $4(8 \%)$ & NS \\
Swelling (\%) & $1(2 \%)$ & $2(4 \%)$ & NS \\
Leg wound infection (\%) & 0 & $1(2 \%)$ & NS \\
Necrosis (\%) & 0 & 0 & NS \\
Leg wound pain (\%) & 0 & $4(8 \%)$ & NS \\
Dysaestesia (\%) & 0 & $4(8 \%)$ & NS \\
\hline
\end{tabular}

NS... not significant $(P>0.05)$

did the swelling, which was higher in the OVH group. The leg-related morbidity decreased significantly one month postoperatively and haematomas disappeared almost completely. The only difference between the groups one month postoperatively was in the incidence of swelling and pain which were lower in the EVH group but the difference was not statistically significant.

As described in the methodology, the sample for histological examination was taken from each harvested vein during the surgery except of four patients where because of lack of suitable vein for grafting no sample was taken. The results of the remaining 96 samples are summarized in Table 5. We can see that 38/96 samples were described as samples with endothelial damage, which is almost $40 \%$ of all samples. We found a statistically significant difference between the EVH and OVH groups in terms of percentage of endothelial damage. Acute endothelial damage was defined as the typical finding of desquamation, granulocyte formation with the presence of fibrin, partly with the formation of tiny defects with bleeding and leukostasis in capillaries of the vasa vasorum. The percentage of acute endothelial damage was higher in the EVH group. Typical acute endothelial damage is shown in Fig. 1, 2.

\section{DISCUSSION}

Open saphenectomy has been the technique of choice for vein harvest in coronary artery bypass surgery for several decades; however, minimally invasive techniques have become increasingly popular in a wide range of surgical specialties. Endoscopic vein harvest has become increasingly used as an alternative to the open technique. The advantages of this technique published in the literature ${ }^{9}$ includes reduced leg wound complications, postoperative pain, required analgesia and incision length. The disadvantage of the procedure according to the literature is the learning curve ${ }^{9}$, risk of carbon dioxide $\left(\mathrm{CO}_{2}\right)$ embolism during $\mathrm{CO}_{2}$ insufflation ${ }^{10}$ and lack of data regarding the long-term patency rates of veins harvested by the endoscopic technique ${ }^{11}$.

Based on our experience, the learning curve is not the major problem of the endoscopic vein harvest. In our series, the harvest time was almost the same in both groups and all surgeons performing the endoscopic vein harvest were able to adopt this method after a relatively short learning curve.

The most important advantage of the EVH is the reduction of leg-related morbidity ${ }^{12-14}$. In our study we found a statistically significant difference between groups only 
Table 5. Histopatological changes.

\begin{tabular}{lccc}
\hline & EVH group & OVH group & $P$-value \\
\hline No. of samples & 47 & 49 & NS \\
Acute endothelial damage (\%) & $24(51 \%)$ & $14(29 \%)$ & $P<0.001$ \\
\hline
\end{tabular}

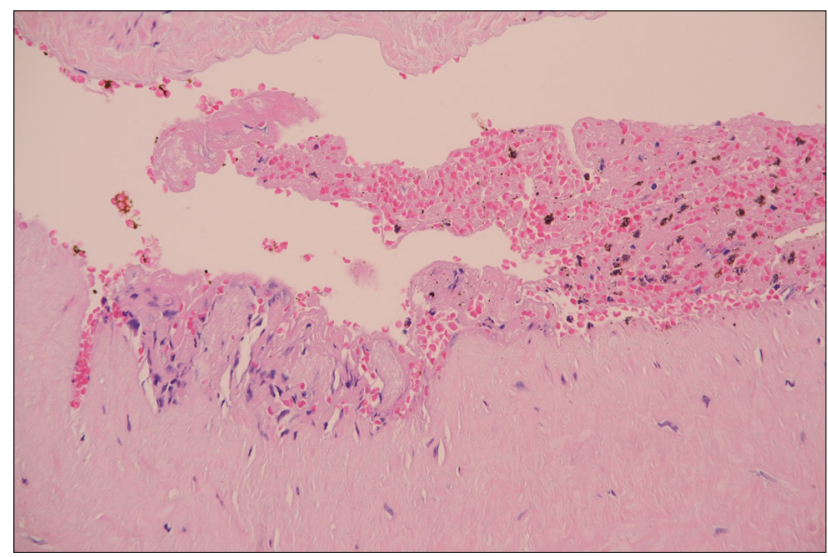

Fig. 1. Acute endothelial damage. Endothelial desquamation with bleeding and fibrin thrombus formation, HE, 400x.

in the postoperative pain 7 days after the surgery. The difference in swelling and dysaesthesia did not reach statistical significance. The reason for the relatively low difference in leg-related morbidity is that generally the incidence of all leg-related mortality in both groups was very low. In spite of that however, we confirmed the superiority of $\mathrm{EVH}$ over $\mathrm{OVH}$ in terms of reduced leg-related morbidity. The only disadvantage of EVH in this field was the relatively high incidence of haematoma in the EVH group. Although the haematoma disappeared in a relatively short time, we recommend using drainage to reduce its incidence.

One major limitation of the current literature is that there is very little conclusive data regarding long-term patency rates of endoscopically harvested veins. There are some concerns that endoscopic vein harvest could be connected with a detrimental effect on vein endothelium which could promote a thrombogenic environment leading to a decrease in graft patency (Rousou et al. ${ }^{15}$ and Zenati et al. ${ }^{16}$ ).

In our study, we found a statistically significant difference between the EVH and OVH groups in terms of percentage of endothelial damage which was statistically significantly higher in the EVH group. Thus we can conclude that there is a difference between the vein harvested endoscopically and the vein harvested by the open technique in terms of the histological picture of the vein. This is also supported by the fact that in our study the pathologist was able to correctly recognize the vein

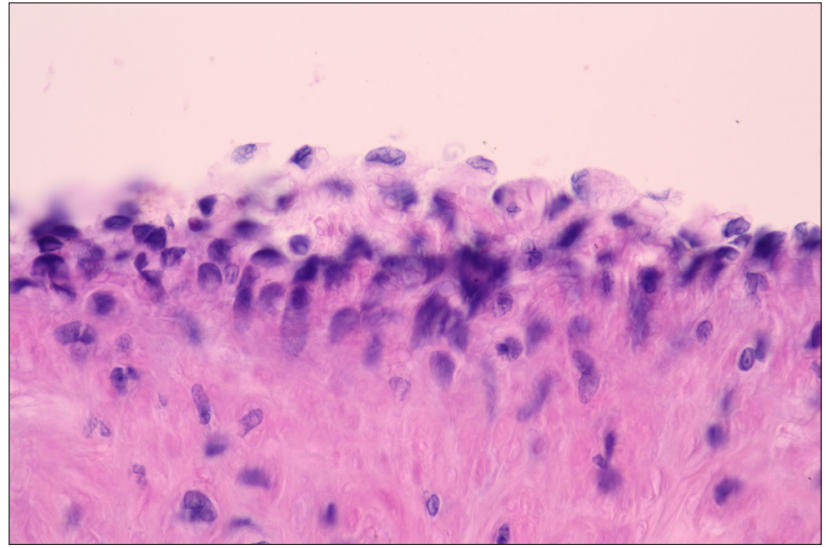

Fig. 2. Acute endothelial damage. Endothelial desquamation, HE, 1000x.

harvested endoscopically in more than $70 \%$ of the cases only according to the histological examination.

\section{CONCLUSIONS}

Endoscopic vein harvest has become increasingly used as an alternative to the open technique. Our study confirmed the advantage of this method in terms of legrelated morbidity as well as cosmetic effect. On the other hand, we found significant histological difference between the vein harvested endoscopically and the vein harvested by the open technique showing that the EVH could be associated with higher acute endothelial damage of the graft. These results support concerns that endoscopic vein harvest could be connected with a detrimental effect on vein endothelium, which may promote a thrombogenic environment leading to a decrease in graft patency, an extremely important factor. That said, the overall incidence of endothelial damage in both groups was very high. The reason for that could be that we detected minor damage which would not influence the long-term patency of the graft. Based on these facts we feel that further investigation of the long-term patency of vein grafts harvested endoscopically is required. 


\section{ACKNOWLEDGEMENT}

This study was supported by the grant of the Internal Grant Agency Ministry of Health Czech Republic, No. NS/10611-3.

\section{REFERENCES}

1. Caparrelli DJ, Ghazoul M, Diethrich EB. Indications for coronary Artery bypass grafting in 2009: what is left to surgery. J Cardiovasc Surg (Torino) 2009;50:19-28.

2. Allen K, Cheng D, Cohn W, Connolly M, Edgerton J, Falk V, Martin J,Ohtsuka T, Vitali R. Endoscopic Vascular Harvest in Coronary Artery Bypass Grafting Surgery: A Konsensus Statement of the International Society of Minimally Invasive Cardiothoracic Surgery (ISMICS) 2005 Innovations: Technology and Techniques in Cardiothoracic and Vascular Surgery 2005,1(2):51-60.

3. Carpino PA, Khabbaz KR, Bojar RM, Rastegar H, Warner KG, Murphy $\mathrm{RE}$, Payne DD. Clinical benefits of endoscopic vein harvesting in patiens with risk factors for saphenectomy wound infections undergoing coronary artery bypass grafting. J Thorac Cardiovasc Surg 2000,119(1):69-75.

4. Reed JF. Leg wound infections following greater saphenous vein harvesting: minimally invasive vein harvesting versus conventional vein harvesting. Int J Low Extrem Wounds 2008,7(4):210-9.

5. Allen KB, Heimansohn DA, Robison RJ, Schier JJ, Griffith GL, Fitzgerald EB, Isch JH, Abraham S, Shaar CJ. Risk factors for leg wound complications following endoscopic versus tranditional saphenous vein harvesting. Heart Surg Forum 2000;3:325-30.

6. Dusterhoft V, Bauer M, Buz S, Schaumann B, Hetzer R. Wound-healing disturbances after vein harvesting for CABG: a randomized trial to compare the minimally invasive direct vision and traditional approaches. Ann Thorac Surg 2001;72:2038-43.
7. Alrawi SJ, Raju R, Alshkaki G, Acinapura AJ, Cunningham JN. Saphenous vein endothelial cell viability: a comparative study of endoscopic and open saphenectomy for coronary Artury bypass grafting. JSLS 2001,5(1):37-45.

8. Rousou LJ, Taylor KB, Lu X, Healey N, Crittenden MD, Khuri SF, Thatte HS. Saphenous vein conduits harvested by endoscopic technique exhibit structural and functional damage. Ann Thorac Surg 2009,87(1):62-70.

9. Markar SR, Kutty R, Edmonds L, Sadat U, Nair S. A meta-analysis of minimally invasive versus traditional open vein harvest technique for coronary artery bypass graft surgery. Interact CardioVasc Thorac Surg 2010;10:266-70.

10. Lin TY, Chiu KM, Wang MJ, Chu SH. Carbon dioxide embolism during endoscopic saphenous vein harvesting in coronary artery bypass surgery. J Thorac Cardiovasc Surg 2003;126:2011-5.

11. Pranjal D, Soroosh $K$, Nannan T. Impact of the learning curve for endoscopic vein harvest on conduit quality and early graft patency. Ann Thorac Surg 2011,91(5):1385-92.

12. Andreasen J, Nekrasas V, Dethlefsen C. Endoscopic vs open saphenous vein harvest for coronary artery bypass grafting: a prospective randomized trial. Eur J Cardiothorac Surg 2008;34:384-9.

13. Ouzounian M, Hassan A, Buth KJ, MacPherson C, Ali IM, Hirsch GM, Ali IS. Impact of Endoscopic Versus Open Saphenous Vein Harvest Techniques on Outcomes After Coronary Artery Bypass Grafting. The Annals of Thoracic Surgery 2010,89(2):403-8.

14. Simek M, Bruk V, Nemec P. Endoskopický odběr vena saphena magna pro revaskularizaci myokardu. Rozhl Chir 2006;85:211-5.

15. Rousou LJ, Taylor KB, Lu XG, Healey N, Crittenden MD, Khuri SF Thatte HS. Saphenous vein conduits harvested by endoscopic technique exhibit structural and functional damage. Ann Thorac Surg 2009;87:62-70.

16. Zenati M, Shroyer L, Collins J. Impact of endoscopic versus open saphenous vein harvest technique on late coronary artery bypass grafting patient outcomes in the ROOBY (Randomized On/Off Bypass) trial. J Thorac Cardiovasc Surg 2011;141: 338-44. 